\title{
ELABORACIÓN DE CONSERVAS A BASE DE COLITAS DE CAMARON Cryphiops Caementarius EN ACEITE AROMATIZADO
}

Responsable: Ing. Luis A. Rivera Chipana Miembro: Ing. Freddy Delgado Cabrera

\begin{abstract}
RESUMEN
El presente trabajo de investigación fue realizado en el Centro de Producción de Tecnología Pesquera (CEPROTEP) de la Facultad de Ingeniería Pesquera, Universidad Nacional JBG Tacna y tiene por finalidad aprovechar el recurso camarón de río (Chryphiops caementarius) para darle un valor agregado.

Para el desarrollo del trabajo, se realizaron seis pruebas experimentales para establecer un flujo adecuado (tratamiento primario, lavado, precocción, envasado, evacuado, esterilizado, enfriado, y almacenamiento).

El análisis sensorial y las pruebas de aceptabilidad fueron realizados con el software Sistema de Análisis Estadistico (S.A.S.).
\end{abstract}

\begin{abstract}
ABSTRAC
The present work of investigation was made in center of production of fishing technology (CEPROTEP) of the Faculty of Fishing Engineering, University National JBG-TACNA and has by purpose of taking advantage of the resource shrimp river (Chryphios caementarius) to give a added value him.
\end{abstract}

For the development of the work, six experimental tests were made to system a suitable flow (primary treatment, washing, cooking, packaging, evacuee, sterilized, cooled and storage).

The sensorial analysis and tests the of acceptability were made with software SAS.

\section{INTRODUCCIÓN}

El problema de la alimentación mundial y la correcta relación entre una buena nutrición-salud es un reto que tienen que solucionar los paises pobres y en vías de desarrollo.

Asimismo cabe indicar que la alimentación mundial va cambiando dia a dia, económicamente a la materia prima se le va dando cada vez mayor valor agregado con el fin de llegar a los mercados exigentes, con productos de alta calidad alimenticia. En tal sentido es que se realizó el presente trabajo. Este se realizó con la finalidad de obtener un producto con cualidades que puedan satisfacer las necesidades de un mercado exigente, tanto nacional como internacional.

\section{OBJETIVOS}

Los objetivos del presente trabajo fueron:

1. Determinar el tiempo de precocido de las colitas decamarón.

2. Evaluar las características físicas, quimicas y microbiológicas de la materia prima y del producto final.

3. Hallar la proporción y tipo adecuado entre líquido de gobierno y materia prima.

4. Determinar el tratamiento térmico de la materia prima.

5. Obtener y optimizar los parámetros de procesamiento para este tipo de conserva.

\subsection{Importancia Económica del Recurso en} la Región Tacna

Este crustáceo es de gran importancia económica y sustenta la pesquería continental de la costa sur del Perú, con capturas de 98 a 356 TM de 1993 al 2000 respectivamente (RODENAS, 2003).

- En la región Tacna las estadísticas de captura de camarón de río son las siguientes:

Según la fuente de PRODUCE-TACNA 2005. indica, año 1996 - 0,40 TM, 1997- 3,03 TM, $1998-2,27$ TM, $1999-1,46$ TM, $2000-3,77$ TM, 2001 - 3,00 TM 2002 - 1,60 TM -2003 $3,30 \mathrm{TM}$, y el año 2004 fue de 5,90 TM.

\section{METOdOLOGÍA}

Para desarrollar el presente trabajo se realizaron seis pruebas experimentales para establecer un flujo adecuado (tratamiento 
primario, lavado, precocción, envasado, evacuado, esterilizado, enfriado y almacenamiento), poniendo énfasis en la determinación en los diferentes controles y resultados.

\section{MATERIALESYMÉTODOS}

\subsection{Lugar de Ejecución}

El presente trabajo de investigación se ha realizado en:

- El Centro de Producción de Tecnología Pesquera (CEPROTEP) de la FAIP/UNJBG.

- El Laboratorio de Tecnología Pesquera de la FAIP / UNJBG.

- El Laboratorio de Microbiología de la FACI / UNJBG.

\section{RESULTADOS Y DISCUSIÓN}

\subsection{De la Materia Prima}

\subsubsection{Análisis Físico Organoléptico.}

\section{a) Características:}

Apariencia General Externa. El camarón de río fue adquirido en el Mercado Grau. Estos tenian un color verde oscuro y con su respectivo brillo. Su textura fue suave y lisa.

Apariencia General Interna. Las vísceras se encontraron intactas, se pudo diferenciar sus partes.

\section{b) Morfologia.}

Los experimentos fueron realizados en el segundo trimestres del año 2005 en épocas en que el camarón estaba todavia en la etapa reproductiva y era escaso; como consecuencia de su escasez, las medidas tomadas a las muestras son las siguientes : tuvieron como longitud promedio de $9,25 \mathrm{~cm}$, ancho $1,55 \mathrm{~cm}$, altura $1,85 \mathrm{~cm}$ y un peso promedio de $21 \mathrm{~g}$.

\subsubsection{Análisis Químico Proximal de la Materia Prima.}

CUADRO N 1 Análisis Químico Proximal de la Materia Prima en Promedio

\begin{tabular}{|l|c|c|}
\hline Componente & $\begin{array}{c}\text { Promedio Colitas } \\
\text { Frescas (\%) }\end{array}$ & $\begin{array}{c}\text { Promedio Colitas } \\
\text { en Conserva (\%) }\end{array}$ \\
\hline Humedad & 83,72 & 84,34 \\
\hline Grasas & 0,7 & 0,65 \\
\hline Proteinas & 14,08 & 13,8 \\
\hline Cenizas & 1,1 & 1,07 \\
\hline Carbohidratios & 0,4 & 0,14 \\
\hline
\end{tabular}

Según el CUADRO $\mathrm{N}^{\circ} 1$, los resultados para humedad van desde 83,72 a $84,34 \%$ por acción de los polifosfatos, los cuales son responsables de la ganancia de humedad; la disminución del porcentaje de proteínas en la conserva de 14,08 a $13,8 \%$; esto se debe al efecto del tratamiento térmico; es decir, por acción de la esterilización existe un desordenamiento de las estructuras y de las cadenas de aminoácidos, en las cuales muchos de estos aminoácidos se convierten en libres y se unen a líquido de gobierno.

La grasa disminuye de las colitas frescas a las colitas en conserva de $0,7 \%$ a $0,65 \%$ por efecto del tratamiento térmico, lo mismo sucede para los carbohidratos de 0,4 a $1,14 \%$; es decir, el tratamiento térmico tiene una acción determinante al eliminar parte de los lípidos en las colitas frescas, así como la pérdida del glucógeno del músculo del camarón. ITP, 2005.

GRÁFICO No 1. Temperatura Interna de la Conserva Vs Temperatura de la Retorta

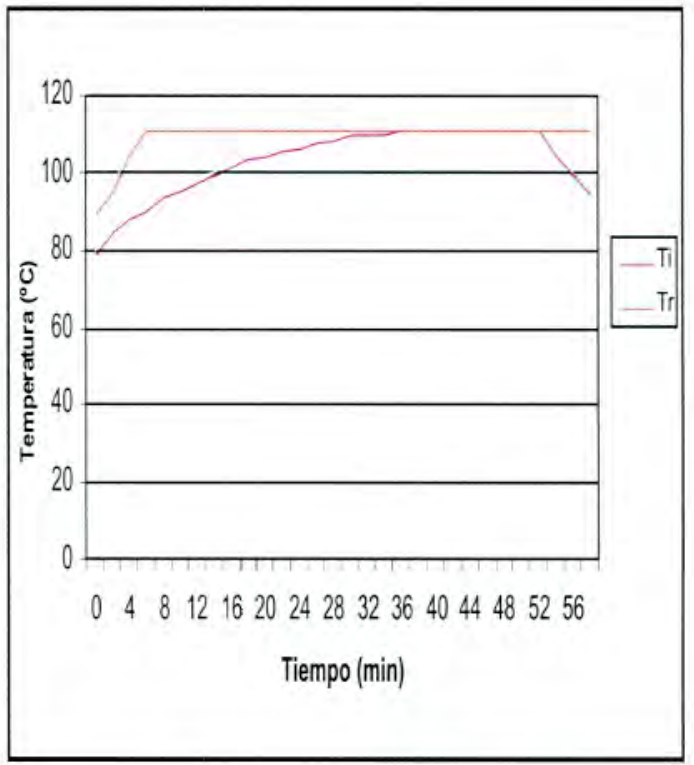

En el Gráfico $\mathbf{N}^{\circ} 1$ se aprecia la temperatura interna ( $\mathrm{Ti})$ de la conserva versus la temperatura de la retorta ( $\mathrm{Tr})$; se muestra cómo va incrementando la temperatura interna de la conserva hasta llegar muy cerca de la temperatura de la retorta, los mismos que tienden a juntarse a partir de los 35 min para después a partir de los 50 min separarse abruptamente en el enfriamiento.

La conserva por estar compuesta de materia sólida y líquida nos muestra una curva quebrada. 
DIAGRAMA N ${ }^{\circ} 1$ BALANCE DE MATERIA

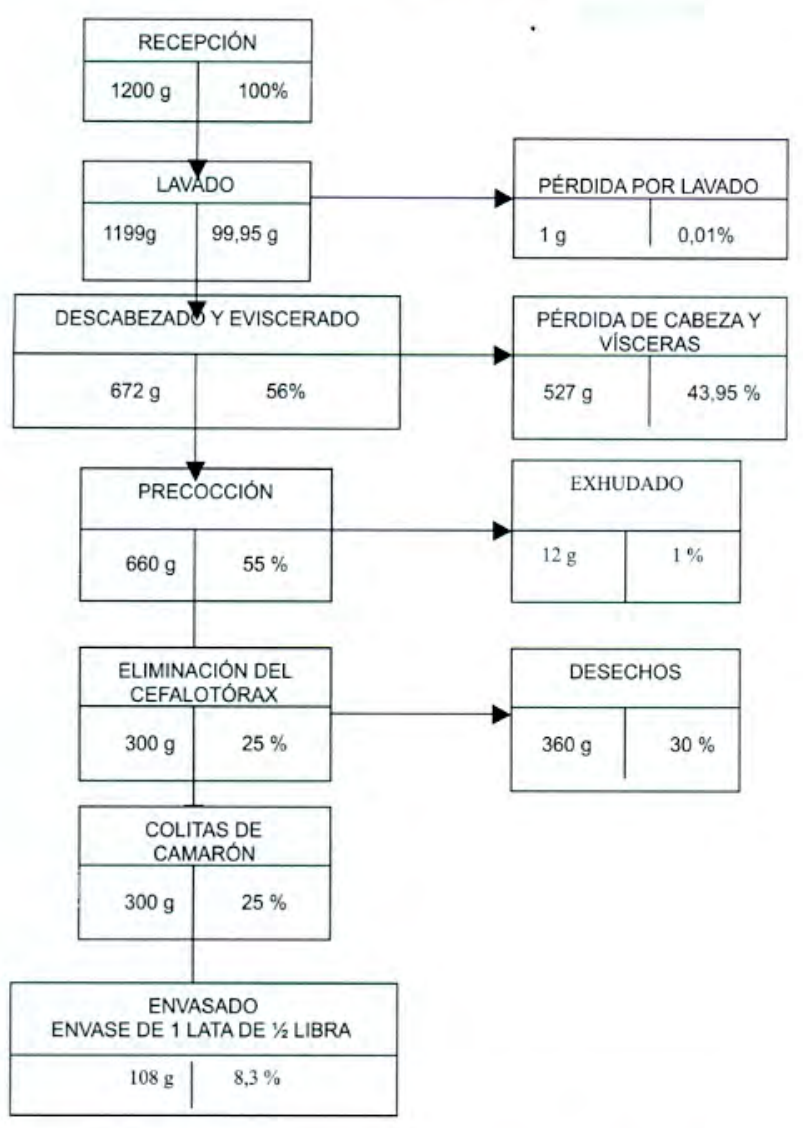

En el Diagrama $N^{\circ} 1$ se muestra el balance de la materia prima, desde la recepción, iniciado en la etapa de adquisición hasta antes del envasado en latas de media libra tipo tuna.

\subsection{Costo de Producción:}

Para acceder a los costos reales de esta conserva se tendrá que emplear los valores del Cuadro $\mathrm{N}^{\circ} 2$

CUADRO No 2. Costo de la Conserva

\begin{tabular}{|c|c|c|c|}
\hline INGREDIENTES & $\begin{array}{l}\text { Pesollata } \\
\text { (G) }\end{array}$ & $\begin{array}{c}\text { Costo por lata } \\
\text { (s.) }\end{array}$ & $\begin{array}{c}\text { Costo por caja } \\
\text { (s.) }\end{array}$ \\
\hline Camarón (colitias) & 108 & 4,66 & 223,68 \\
\hline Lia.de gobierno & 72 & & \\
\hline $\operatorname{EDTA}(0,1 \%)$ & 0,08 & 0,00084 & 0,04032 \\
\hline Polifosiátos $(0,2 \%)$ & 0,16 & 0,00112 & 0,05376 \\
\hline $\mathrm{Sal}(2.5 \%)$ & 2 & 0,0008 & 0,0384 \\
\hline Ac. Citrico $(0.01 \%)$ & 0,008 & 0,00012 & 0,00576 \\
\hline \multirow[t]{2}{*}{$\operatorname{GNS}(0,05 \%)$} & 0,04 & 0,0006 & 0,0288 \\
\hline & & 0,00348 & 0,16704 \\
\hline Alquiler de planta & & 0,1 & 4,8 \\
\hline Envase & & 0,3838 & 18,4224 \\
\hline Subtotal & & 5,14728 & 247,069 \\
\hline Combustible $(\$ 0,4)$ & & 0,0279 & 1,34 \\
\hline Costo administrafivo $(1 \%)$ & & 0,051 & 2,4706 \\
\hline $\begin{array}{l}\text { Costo de mano de obra } \\
(10 \%)\end{array}$ & & 0.514 & 24.7069 \\
\hline Impuesto (18\%) & & 0,997 & 48,9779 \\
\hline Imprevistos (5\%) & & 0,2573 & 12,354 \\
\hline TOTAL & & 6,976 & 336,9185 \\
\hline
\end{tabular}

Del cuadro anterior se observa que el mayor costo es de la materia prima, el mismo que puede reducirse si se realiza un cultivo intensivo y en épocas de cosecha; el resto de los costos son los que enfrenta toda empresa privada; por lo tanto, poco podria disminuir.

El costo de producción de cada lata de conserva de colitas de camarón de $1 / 2$ libra tipo tuna es de S/. 6,97 y el costo por caja de 48 unidades es de S/. 336,91; el precio es elevado para el mercado local; por eso, esta conserva no estaría orientada para satisfacer la necesidades proteicas de nuestra población, más bien esta conserva es un producto "delicatessen" orientado en nuestro país a un segmento social que tenga el suficiente poder económico o para consumirlos en mercados internacionales.

\section{CONCLUSIONES}

1. La proporción adecuada entre sólidos y líquido de gobierno es de $60 \%$ y $40 \%$.

2. El pH para las colitas de camarón en conserva fue de 8,09 y para el líquido de gobierno de 7,58.

3. El flujo de los parámetros de tecnológicos del procesamiento de la conserva es: tratamiento primario de la materia prima, descabezado y eviscerado, lavado, precocción (6 min ), envasado (envases de hojalata de $1 / 2$ libra), adición del líquido de gobierno, evacuado $\left(90^{\circ} \mathrm{C}\right.$ por $5 \mathrm{~min})$, sellado, esterilizado $\left(112^{\circ} \mathrm{C}\right.$ por 41,8 min y un F0 de 3,95), almacenado (temperatura ambiente).

4. La composición química general del producto: humedad $84,34 \%$; grasas $0,65 \%$; proteínas $13,8 \%$; cenizas $1,07 \%$ y carbohidratos $0,14 \%$.

5. El rendimiento final del producto es de $25 \%$

6. La conserva es aceptable desde el punto de vista químico, físico, orgaroléptico, microbiológico y sensorial.

\section{RECOMENDACIONES}

- Realizar el envasado con otro tipo de líquido de gobierno dependiendo del tipo de mercado al que va dirigido.

- Utilizar para el producto envases de aluminio de $87 \mathrm{ml}$ y $103 \mathrm{ml}$ tipo "ring-pull easy-open body".

- Tener mucho cuidado con este tipo de productos especiales al momento de determinar el punto más frío, pues la forma de transmisión de calor es por conducción y convección.

- Para determinar el Fo y el tiempo de procesamiento se recomienda utilizar el método de Ball, el cual refleja resultados más exactos. 


\section{BIBLIOGRAFÍA}

Ángeles, J. (1972). Estudio taxonómico comparativo entre el Cryphiops caementarius (Molina 1872) y el Macrobrachium inca (Holthuis, 1950) de nuestros puertos costeros. U.N.F.V. Lima.

Anzaldua (1994). Evaluación sensorial de los alimentos en la teoría y practica. Barcelona.

A.O.A.C. Association of Official And agriculture Chemist Methods of Analisys (1990). 14th Ed., Washington D.C.

Ariansen, C. (1990). Ciencia y tecnología de los alimentos. Universidad de Lima, Facultad de Ingeniería Industrial, Lima .

Bertullo, H. (1975). Tecnología de los productos y sub productos del pescado, moluscos y crustáceos. Editorial Hemisferio Sur, Buenos Aires.
Bettison, J (1994). Procesado térmico y envasado de los alimentos .Edición Acribia S.A, Edición en español. Zaragoza, España.

Calzada, J. (1984). Métodos estadisticos para la investigación." 6ta edición. Editorial Milagros S.A. Lima, Perú.

Carranza, R. (1987). Determinación del tratamiento térmico. Traducción de Ranganna. FAIP/UNJBG, Tacna, Perú.

Instituto Tecnológico Perú / Agencia de Cooperación Internacional del Japón (1997). "Tecnología de Procesamiento de Productos Pesqueros". IX Curso Internacional ITP, Lima . Instituto Tecnológico Perú / Agencia De Cooperación Internacional

del Japón (1999). "Tecnología de Procesamiento de Productos Pesqueros". XII Curso Internacional ITP, Lima. 